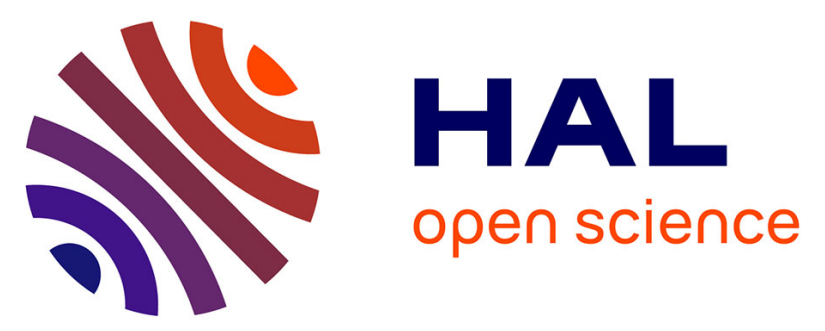

\title{
Versatile synthesis of alpha-fused BODIPY displaying intense absorption in the NIR region and high electron affinity
}

Quentin Huaulme, Alexandra Sutter, Sadiara Fall, Denis Jacquemin, Patrick

Leveque, Pascal Retailleau, Gilles Ulrich, Nicolas Leclerc

\section{To cite this version:}

Quentin Huaulme, Alexandra Sutter, Sadiara Fall, Denis Jacquemin, Patrick Leveque, et al.. Versatile synthesis of alpha-fused BODIPY displaying intense absorption in the NIR region and high electron affinity. Journal of Materials Chemistry C, 2018, 6 (37), pp.9925-9931. 10.1039/c8tc03138j . hal02140883

\section{HAL Id: hal-02140883 \\ https://hal.science/hal-02140883}

Submitted on 5 Jan 2022

HAL is a multi-disciplinary open access archive for the deposit and dissemination of scientific research documents, whether they are published or not. The documents may come from teaching and research institutions in France or abroad, or from public or private research centers.
L'archive ouverte pluridisciplinaire HAL, est destinée au dépôt et à la diffusion de documents scientifiques de niveau recherche, publiés ou non, émanant des établissements d'enseignement et de recherche français ou étrangers, des laboratoires publics ou privés. 


\section{Journal of \\ Materials Chemistry C}

\section{Accepted Manuscript}

This article can be cited before page numbers have been issued, to do this please use: Q. Huaulmé, A. Sutter, S. Fall, D. Jacquemin, P. Lévêque, P. Retailleau, G. Ulrich and N. Leclerc, J. Mater. Chem. C, 2018,

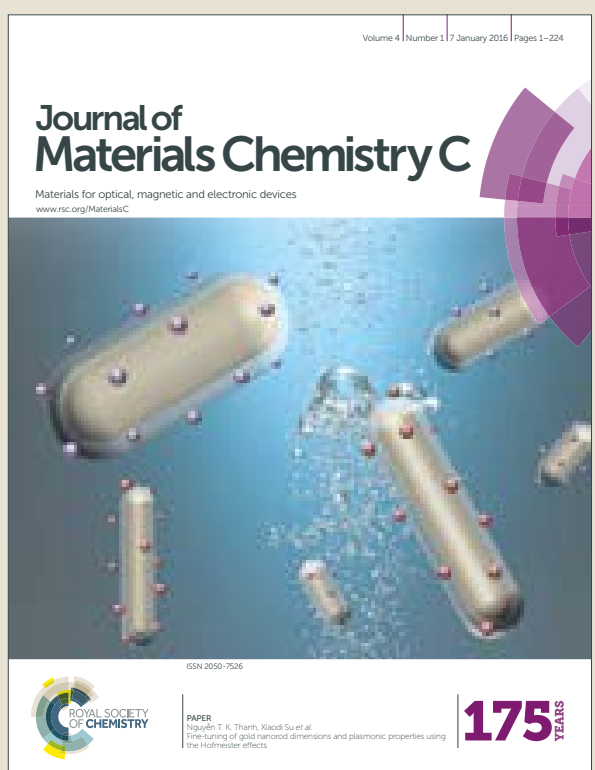

This is an Accepted Manuscript, which has been through the Royal Society of Chemistry peer review process and has been accepted for publication.

Accepted Manuscripts are published online shortly after acceptance, before technical editing, formatting and proof reading. Using this free service, authors can make their results available to the community, in citable form, before we publish the edited article. We will replace this Accepted Manuscript with the edited and formatted Advance Article as soon as it is available.

You can find more information about Accepted Manuscripts in the author guidelines.

Please note that technical editing may introduce minor changes to the text and/or graphics, which may alter content. The journal's standard Terms \& Conditions and the ethical guidelines, outlined in our author and reviewer resource centre, still apply. In no event shall the Royal Society of Chemistry be held responsible for any errors or omissions in this Accepted Manuscript or any consequences arising from the use of any information it contains. 


\title{
Versatile synthesis of $\alpha$-fused BODIPY displaying intense absorption in the NIR and high electron affinity
}

Received 00th January 20xx, Accepted 00th January $20 x x$

DOI: $10.1039 / \times 0 \times x 00000 x$

www.rsc.org/

\author{
Quentin Huaulmé, ${ }^{\mathrm{a}}$ Alexandra Sutter, ${ }^{\text {a }}$ Sadiara Fall, ${ }^{\mathrm{b}}$ Denis Jacquemin, ${ }^{* c}$ Patrick Lévêque, ${ }^{\mathrm{b}}$ Pascal \\ Retailleau, ${ }^{\text {d }}$ Gilles Ulrich*a and Nicolas Leclerc*a
}

\begin{abstract}
We report the design and the highly efficient and versatile synthesis of four isomers of $\alpha$-fused boron dipyrromethene (BODIPY). Structure-property relationships have been established through photophysical and electrochemical properties analysis as well as first-principle calculations. A comparative study of their charge transport properties has been carried out in Organic Field Effect Transistor (OFET) devices, and allowed the identification a high mobility material $\left(10^{-2} \mathrm{~cm}^{2} . \mathrm{V}^{-1} . \mathrm{s}^{-}\right.$ $\left.{ }^{1}\right)$ displaying a balanced ambipolar behavior.
\end{abstract}

\section{Introduction}

The continuous development of dyes for organic semiconducting materials, including optoelectronic devices, bioelectronics, chemical sensors, and biological labelling, has led to the elaboration of a large and diverse library of molecular scaffolds. Among them, boron dipyrromethene (BODIPY) represents an extraordinary class of fluorophores, displaying large molar absorption coefficients, high fluorescence quantum yields and valuable chemical and photochemical stabilities. ${ }^{1}$ In addition, BODIPY derivatives display numerous possibilities of functionalization, allowing the fine-tuning of their physico-chemical properties through structural variations. Consequently, they have been used in various fields, e.g. laser dyes, ${ }^{2}$ bio-imaging materials, ${ }^{3}$ or organic solar cells. ${ }^{4}$ Recently, several studies have shown that BODIPY cores could be considered as chemical platforms for the elaboration of extended polycyclic aromatic units by fusing rigid aromatic units either in their meso-position, ${ }^{5}$ ß-position, ${ }^{6}$ or $\alpha$-position. ${ }^{7}$ Polycyclic aromatic units have emerged as key players for molecular electronic applications thanks to their extended $\pi$-delocalization combined with potentially favourable supramolecular organizations. ${ }^{8}$ Moreover, they often exhibit more-intense Near Infra-Red (NIR) absorption and emission. In that context, $\alpha$-fused BODIPY and azaBODIPY dyes have attracted interest from several groups. ${ }^{7,9}$ As often in the synthesis approaches used in polycyclic aromatic molecules, the ring closure by oxidative coupling is a key step, but it allows only a limited control over the regioselectivity of the reaction. Consequently, these highly interesting molecules suffer from a lack of versatility in their synthesis. Herein, we report the very straightforward, effective and versatile synthesis of four new dithienyl $\alpha$-fused BODIPY isomers. The preparation of these compounds answers two synthetic challenges: i) the regioselective tetra-functionalization of the 2, 3, 5 and 6 positions of an unsubstituted BODIPY core by halogenation and Stille cross-coupling reactions and, in a second step, ii) the regioselective intramolecular oxidative ring closure reaction between two vicinal thiophene substituents (see Scheme 1). Their complete characterizations via NOESY 2D NMR, X-ray diffraction of single crystals, optical spectroscopies in solution and in solid state, first principle calculations and the preliminary investigation of their hole and electron charge transport properties allowed us to demonstrate and rationalize the deep impact of chemical regioselectivity on the optoelectronic properties of these promising BODIPY-based polycyclic aromatic units.

\section{Results and Discussion}

\section{Synthesis.}

c. CEISAM, UMR CNRS-6230, BP 92208, 2 rue de la Houssinière, 44322 Nantes, Cedex 03, France. *Email: Denis.Jacquemin@univ-nantes.fr

d. Institut de Chimie des Substances Naturelles, CNRS-UPR2301, Université ParisSaclay, Bât. 27-1 avenue de la Terrasse, 91198 Gif-sur-Yvette Cedex, France Electronic Supplementary Information (ESI) available: See DOI: $10.1039 / x 0 x \times 00000 x$

See




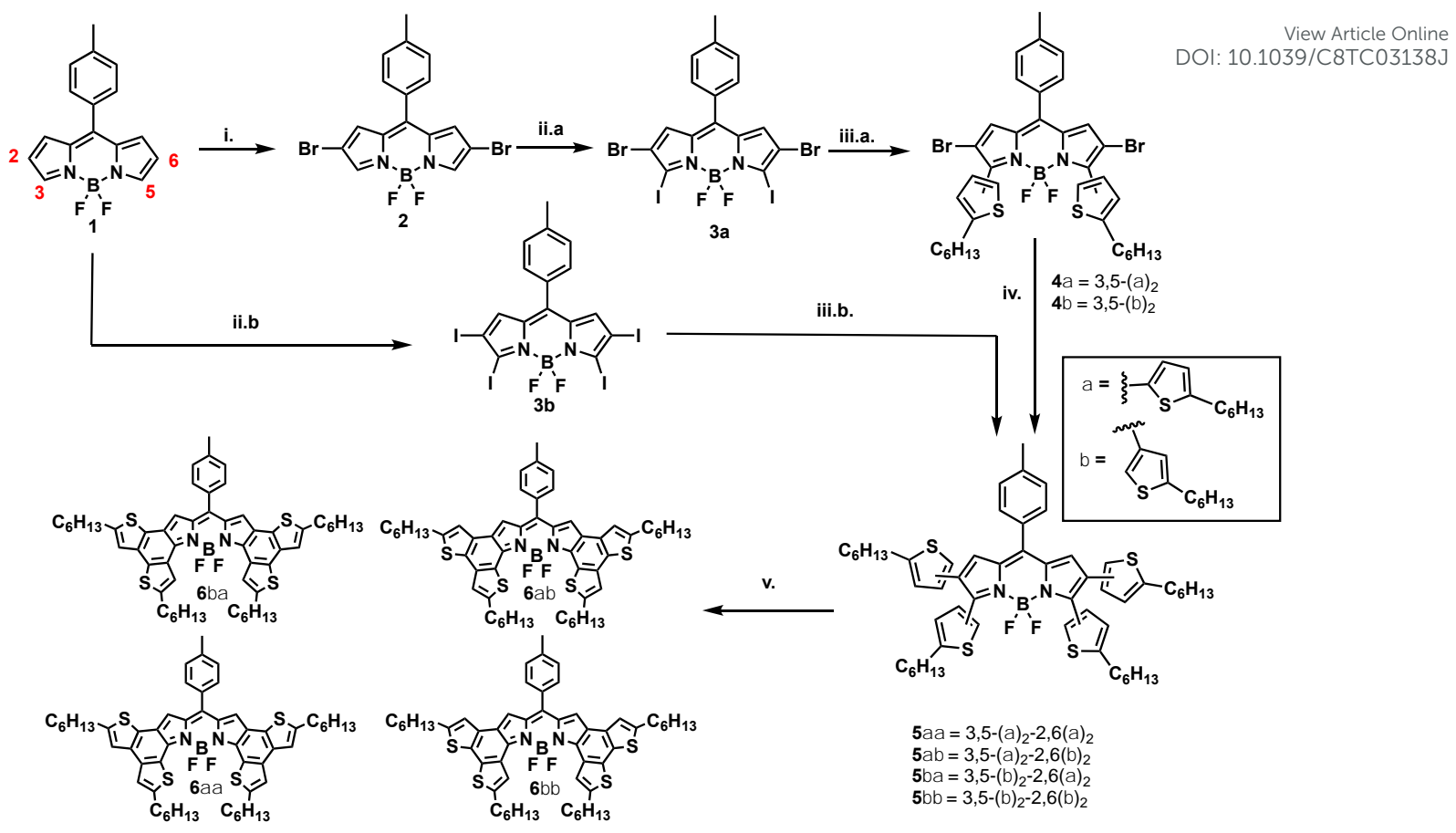

Scheme 1 Synthetic route for the targeted $\alpha$-fused BODIPY (see the ESI for detailed synthetic procedures and analysis). Keys: i. NBS, $\mathrm{CHCl}_{3} / \mathrm{DMF}, 80 \%$; ii. $\mathrm{I}_{2}, \mathrm{HIO}_{3}, \mathrm{EtOH} / \mathrm{H}_{2} \mathrm{O}, 66 \%$ (a. 2.5 eq; b. 5 eq); iii. T⿱1$-\mathrm{SnBu}_{3}$ (a. 2.1 eq; b. 4 eq), $\left[\mathrm{Pd}\left(\mathrm{PPh}_{3}\right)_{4}\right] 68-80 \%$; iv. $\mathrm{T}^{2}-\mathrm{SnBu}_{3},\left[\mathrm{Pd}\left(\mathrm{PPh}_{3}\right)_{4}\right]$, $\mathrm{PhMe}, 79-84 \%$; v. $\mathrm{FeCl}_{3}$, DCM.

The synthesis of all the tetra-functionalized BODIPY derivatives described in the literature required four synthetic steps, including a halogenation step of the unstable BODIPY dipyrrin precursor. ${ }^{10}$ The synthetic route that we disclose here not only reduces the number of synthetic steps required, but also avoids handling any unstable precursor. The unsubstituted BODIPY $\mathbf{1}$ and $\mathbf{2}$ were obtained following reported procedures. $^{7}$ The key intermediate 3a was designed in order to induce a high regioselectivity in the following Stille crosscoupling. Although Erhong et al. recently demonstrated the higher reactivity of the 3 and 5 positions of the tetrabrominated BODIPY core compared to its 2 and 6 positions toward the oxidative addition of a palladium(0) complex, ${ }^{11}$ we nevertheless decided to introduce an iodine atom in its 3 and 5 positions to further increase the difference of reactivity during the Stille cross-coupling. Inspired by a previous work, ${ }^{12}$ the iodation has been performed using $\mathrm{I}_{2} / \mathrm{HIO}_{3}$ in a highly dissociative solvent mixture in relatively good yield. The regioselectivity of this second halogenation step has been unambiguously determined by NOESY 2D-NMR experiment, which clearly indicates a coupling through space between the remaining protons of the BODIPY core and one of the $A B$ system of the tolyl residue (see Figure S49). Compound $\mathbf{3 b}$ designed for the synthesis of tetra-homosubstituted derivatives was obtained using the same condition and 4.5 equivalents of both $\mathrm{I}_{2}$ and $\mathrm{HIO}_{3}$ with a very good yield (91\%). The di-substituted symmetrical intermediates $4 \alpha$ and $4 \beta$ (bearing the thiophenes on the 3,5-position) were synthetized in very good yields $(60-89 \%)$ by the use of 2 equivalents of stannic derivatives. The tetra-homosubstituted intermediates $\mathbf{5} \boldsymbol{\alpha} \boldsymbol{\alpha}$ and $\mathbf{5} \boldsymbol{\beta} \boldsymbol{\beta}$ were obtained directly from the tetra iodinated
BODIPY 3b in optimized yields $(68-81 \%)$ with $\mathrm{Pd}\left(\mathrm{PPh}_{3}\right)_{4}$ as catalyst and 8 equivalents of tri-butyl-stannyl-thiophene. The compounds $\mathbf{5} \boldsymbol{\alpha} \boldsymbol{\beta}$ and $\mathbf{5} \boldsymbol{\beta} \boldsymbol{\alpha}(\mathrm{T} 1 \neq \mathrm{T} 2)$ were obtained by a second step of Stille coupling starting from $4 \alpha$ or $\mathbf{4} \beta$ with the same synthetic procedure in high yields (79-84\%). In the presence of a stoichiometric amount of iron(III) chloride ( 5 eq) as oxidant, all of the tetra-substituted BODIPY 5 quickly undergo oxidative ring closure to give the corresponding $\alpha$-fused BODIPY 6 in good to excellent yields (63-97\%). For the first time, this strategy allowed the straightforward synthesis of four isomers of boron difluoride-chelating nine fused-rings systems. The molecular structures of all the intermediates and final compounds were attributed by ${ }^{1} \mathrm{H},{ }^{13} \mathrm{C},{ }^{11} \mathrm{~B},{ }^{19} \mathrm{~F} N \mathrm{NMR}$ and $\mathrm{HR}-$ MS. The regioselectivity of the oxidative ring closure has been demonstrated using X-ray diffraction (for two compounds).

\section{X-ray structures.}

X-ray diffraction structures could be obtained for both $6 \beta \alpha$ and $6 \alpha \beta$ lanconfirming the regioisomers that embody two distinct human shapes with a tolyl group as a head put upon a BODIPY core in meso position and four hexyl chains disposed like pairs of arms and legs (see Table S1 in SI)

Compound $\mathbf{6} \boldsymbol{\alpha} \boldsymbol{\beta}$ crystallize in the monoclinic centrosymmetric space group $\mathrm{C} 2 / \mathrm{c}$ and has a crystallographic C2 symmetry (the hexyl legs show disorder that could potentially break the strict symmetry although the disorder was chosen to be treated as static over two atomic sites lengthwise (Figure 1 left)). Furthermore, it displays an approximate non-crystallographic $\mathrm{C}_{2 \mathrm{~h}}$ symmetry with an overall planar - albeit slightly sinusoidal - structure (Figure 1 middle) that 
Table 1 Spectroscopic data recorded in diluted solutions $\left(-10^{-6} \mathrm{M}\right.$, aerated toluene) at $25^{\circ} \mathrm{C}$. a) Quantum yields calculated using the following standards: rhodamine $6 \mathrm{G}$ in water $\left(\Phi_{\mathrm{F}}\right.$

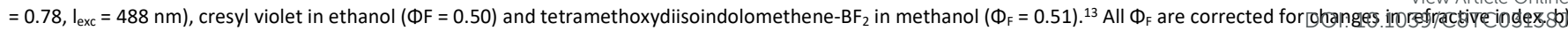
$\mathrm{k}_{\mathrm{r}}$ and $\mathrm{k}_{\mathrm{nr}}$ were calculated using the following equations: $\mathrm{kr}=\Phi_{\mathrm{F}} / \tau, \mathrm{knr}=\left(1-\Phi_{\mathrm{F}}\right) / \tau$ assuming that the excited state is obtained with unit efficiency. The uncertainties on the $I_{\text {max }}, \varepsilon^{\mathrm{lmax}}$ and $Q_{F}$ are $1 \mathrm{~nm}, 5 \%$ and $10 \%$, respectively

\begin{tabular}{ccccccccc}
\hline Compound & $\begin{array}{c}\lambda_{\boldsymbol{a b s}} \\
(\mathrm{nm})\end{array}$ & $\begin{array}{c}\boldsymbol{\varepsilon} \\
\left(10^{5} \mathrm{M}^{-1} \cdot \mathrm{cm}^{-1}\right)\end{array}$ & $\begin{array}{c}\boldsymbol{\lambda}_{\boldsymbol{e m}} \\
(\mathrm{nm})\end{array}$ & $\boldsymbol{\phi}_{\boldsymbol{F}}^{\mathrm{a})}$ & $\begin{array}{c}\boldsymbol{\tau} \\
(\mathrm{ns})\end{array}$ & $\begin{array}{c}\boldsymbol{k}_{\boldsymbol{r}}^{\mathrm{b})} \\
\left(10^{8} \mathrm{~Hz}\right)\end{array}$ & $\begin{array}{c}\left.\boldsymbol{k}_{\boldsymbol{n}} \boldsymbol{b}^{\mathrm{b}}\right) \\
\left(10^{8} \mathrm{~Hz}^{2}\right.\end{array}$ & $\begin{array}{c}\boldsymbol{\Delta}_{\boldsymbol{S S}} \\
\left(\mathrm{cm}^{-1}\right)\end{array}$ \\
\hline $\mathbf{4 \alpha}$ & 639 & 0.612 & 681 & 0.47 & 2.05 & 2.29 & 2.59 & 965 \\
$\mathbf{4} \boldsymbol{\beta}$ & 598 & 0.815 & 630 & 0.45 & 3.56 & 1.26 & 1.54 & 849 \\
$\mathbf{5} \boldsymbol{\alpha} \boldsymbol{\alpha}$ & 664 & 0.487 & 721 & 0.13 & 2.19 & 0.594 & 3.97 & 1191 \\
$\mathbf{5} \boldsymbol{\alpha} \boldsymbol{\beta}$ & 647 & 0.950 & 688 & 0.49 & 1.85 & 2.65 & 2.76 & 921 \\
$\mathbf{5} \boldsymbol{\beta} \boldsymbol{\alpha}$ & 654 & 0.574 & 712 & 0.13 & 2.24 & 0.580 & 3.88 & 1246 \\
$\mathbf{5} \boldsymbol{\beta} \boldsymbol{\beta}$ & 623 & 0.602 & 663 & 0.41 & 2.07 & 1.98 & 2.85 & 968 \\
$\mathbf{6} \boldsymbol{\alpha} \boldsymbol{\alpha}$ & 758 & 1.22 & - & - & - & - & - & - \\
$\mathbf{6} \boldsymbol{\alpha} \boldsymbol{\beta}$ & 719 & 2.29 & 730 & 0.08 & 7.91 & 0.101 & 1.16 & 210 \\
$\mathbf{6} \boldsymbol{\beta} \boldsymbol{\alpha}$ & 665 & 0.758 & - & - & - & - & - & - \\
$\mathbf{6} \boldsymbol{\beta} \boldsymbol{\beta}$ & 674 & 1.41 & - & - & - & - & - & - \\
\hline
\end{tabular}
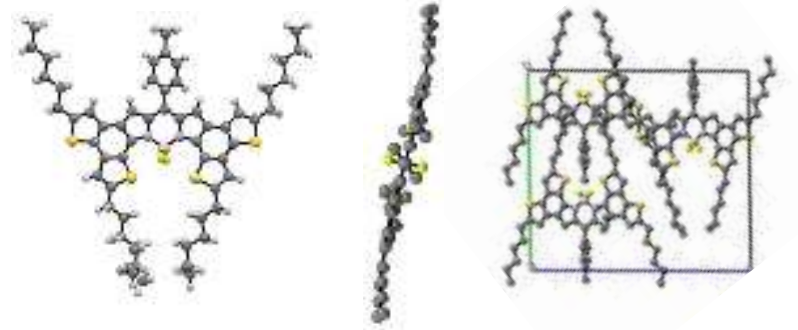

Fig. $1 \mathrm{X}$-Ray structure of compound $\mathbf{6} \boldsymbol{\alpha} \boldsymbol{\beta}$ facial view (left), in plane (middle), headto-tail packing (right).

encompasses the thiophene $\alpha$-fused BODIPY platform, and the hexyl arms (except the terminal C19): the rms deviation of that mean plane is indeed low, $0.113 \AA$, and only fluoride atoms, C19 and the disordered hexyl legs ( $\mathrm{C} 19$ and C5B atoms lie out of the plane at $c a$. $0.4 \AA$ ), and the tolyl group which makes a dihedral angle of $c a .51^{\circ}$ with the central BODIPY core are significantly out of the aforementioned mean plane. This dye with the "hands in the air" features an almost equilateral triangle of maximum length (from "fingers to toes") of $21.3 \AA$, and its symmetry reflects the entire $\pi$-delocalization on the nine fused cycle. It presents typical bond lengths close to the values found for other BODIPY: $N-B=$ $1.557 \AA$ and $B-F=1.384 \AA$, with a geometry around the boron that is tetrahedral with an angle of $110.48^{\circ}$ with the polycyclic skeleton.

In the crystal, all the molecules are oriented along the $b$ direction at position $0, y, 0.25$ with the tolyl residue pointing between the two vicinal hexyl legs but lying in the $\left(\begin{array}{lll}-1 & 0 & 2\end{array}\right)$ plane with interplanar distances of $3.78 \AA$. Despite that assembly in layers, only one
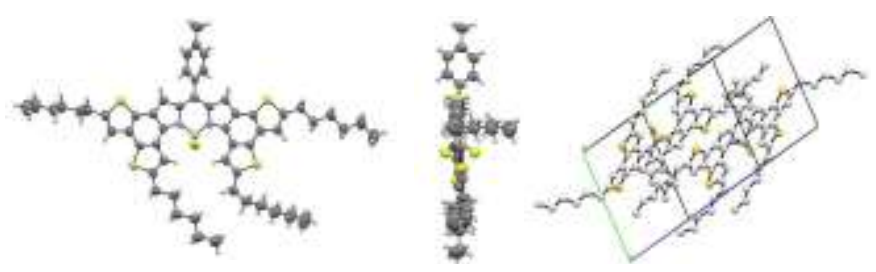

Fig. 2 X-Ray structure of compound $\mathbf{6} \boldsymbol{\beta} \boldsymbol{\alpha}$ facial view (left), in plane (middle), packing (right).

stacking interaction occurs specifically between $\mathrm{T} 2$ thiophene rings at general position and at $3 / 2-x, 3 / 2-y, 1-z$ with $1.58 \AA$ of slippage. Stability between layers is reinforced by anchoring the fluoride atoms with the tolyl methyl at $x-0.5, y-0.5, z$ and the $\mathrm{C} 10 \mathrm{~B} / \mathrm{C}$ methyl at 1.5-x, 0.5-y, 1.5-z. Within the planes, in the other direction, the molecules related by center of inversion are held jointly with their longest edges parallel to each other.

An X-ray crystal structure could be obtained for $\mathbf{6} \boldsymbol{\beta} \boldsymbol{\alpha}$ as well, which again confirms the nature of the regioisomer (Figure 2). Here the hexyl arms are in cross with one arm slightly bent, which decreases the span of the molecule to $c a .28 \AA$. The hexyl legs are not fully stretched either but oriented parallel to the same direction on the side of the curved arm The core of the BODIPY is fully planar over the nine cycles, the tolyl has dihedral angle of $57.71^{\circ}$ with the main core and the boron adopts a tetrahedral geometry. Typical bond lengths are again observed $\mathrm{N}-\mathrm{B}=1.55 \AA$ and $\mathrm{B}-\mathrm{F}=1.39 \AA$. As observed in ${ }^{1} \mathrm{H}$ NMR (through space $\mathrm{H}-\mathrm{F}$ coupling) the fluorine are relatively closed with an equal distance of $2.41 \AA$ to the $\mathrm{H}$ of the two $\mathrm{T}^{1}$ thienyls.

\section{Spectroscopic studies.}




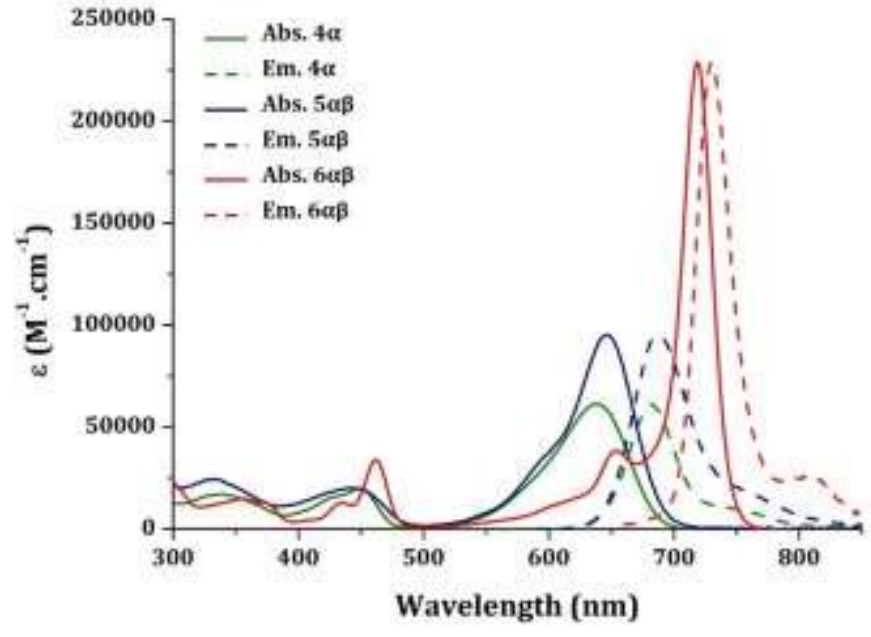

Fig. 3 Absorption spectra and emission spectra of $4 \alpha, 5 \alpha \boldsymbol{\beta}$ and $\mathbf{6} \boldsymbol{\alpha} \boldsymbol{\beta}$ (recorded in toluene at $25^{\circ} \mathrm{C} ; \sim 10^{-6} \mathrm{M}$ )

The spectroscopic data, absorption and emission measurements, of all the synthetized isomers were investigated and are summarized in Table 1.

All $5 \mathbf{T}^{1} \mathbf{T}^{2}$ compounds show strong absorptions in the red region (623-664 nm). By comparing them to $4 \boldsymbol{\alpha}$, one notices that the two supplementary thiophene groups at the 2,6 positions of the BODIPY induce only relatively small bathochromic effects. Moreover, one can notice that due to a better conjugation of thiophene units when grafted in their $\boldsymbol{\alpha}$-positions, $\mathbf{5} \boldsymbol{\alpha} \boldsymbol{\alpha}$ exhibits the highest absorption wavelengths. In addition, the substitutions on the 3,5 positions have the strongest impact on the absorption wavelength as previously observed in thiophene substituted BODIPY. ${ }^{14}$ After cyclisation of compounds 5 to compounds $\mathbf{6}$ the absorption and emission profiles undergo strong bathochromic (100 to $150 \mathrm{~nm}$ ) and hyperchromic shifts. Those effects are attributed to the enhancement of the electronic delocalization all over the molecular backbone induced by the coplanarization of the peripheral substituents and to the stiffening of the structure (Figure 3). The absorption spectra of the four fused compounds in solution are shown in Figure 4. Similarly to their non-fused precursors, stronger bathochromic effects are observed for compound with an $\alpha$ type thiophene on the "3,5-" position of the BODIPY precursor. A very strong enlargement of the absorption peaks is observed for the two dyes bearing a $\beta$ type thiophene on 3,5-position (FWMH going from 484 to $1426 \mathrm{~cm}^{-1}$ for $\mathbf{6} \boldsymbol{\alpha} \boldsymbol{\beta}$ and $\mathbf{6} \boldsymbol{\beta} \boldsymbol{\beta}$, respectively). In the case of $\mathbf{6 \beta \alpha}$ the spectrum becomes extremely broad.

As a consequence, by playing on the selectivity of the grafting position on the thiophene residues and then on the oxidative ring closure positions, it is possible to significantly tune the optical properties along the four isomers series. In comparison to the all $\beta$ thiophene isomer $\mathbf{6} \boldsymbol{\beta} \boldsymbol{\beta}$, the all $\boldsymbol{\alpha}$-thiophene isomer $\mathbf{6} \boldsymbol{\alpha} \boldsymbol{\alpha}$ presents an absorption maxima red-shifted by more than $80 \mathrm{~nm}$, which corresponds to an electronic transition decreased by $c a .0 .2 \mathrm{eV}$.

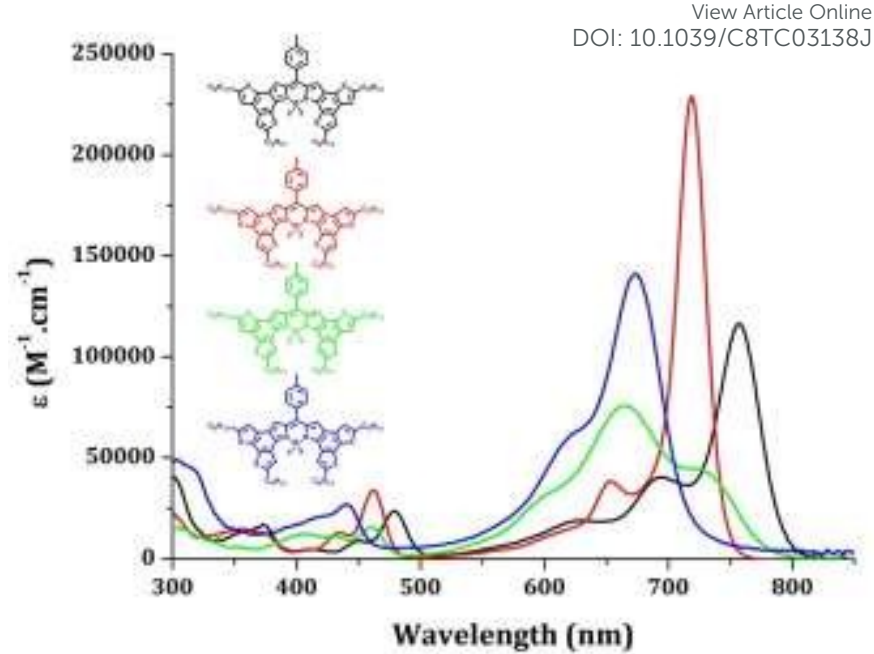

Fig. 4 Absorption spectra of the four $\alpha$-fused BODIPYs $6 \mathrm{~T}^{1} \mathrm{~T}^{2}$ (recorded in toluene at $\left.25^{\circ} \mathrm{C} ; \sim 10^{-6} \mathrm{M}\right)$.

All compounds 5 fluoresce in the red-NIR region (663-721 $\mathrm{nm}$ ). Compounds with a $\beta$-thiophene on 2,6-positions show the lowest emission wavelengths ( 663 and $688 \mathrm{~nm}$ for $\mathbf{5} \boldsymbol{\beta} \boldsymbol{\beta}$ and $\mathbf{5} \boldsymbol{\alpha} \boldsymbol{\beta}$ ) but present high radiative rates. The two other isomers emitting at 710 $721 \mathrm{~nm}$ are less fluorescent, the introduction of $\alpha$-thiophene units on the 2,6-positions seems to severely decrease the radiative rate, which is confirmed by a comparison with $\mathbf{4} \boldsymbol{\alpha}$. After cyclisation, compound $\mathbf{6} \boldsymbol{\alpha} \boldsymbol{\beta}$ remains the only emissive structure in aerated solvent, and it shows a reduced non-radiative deexcitation constant and a much longer lifetime compared to the precursors of type $\mathbf{5}$. This observation is coherent with the increase of the rigidity of the molecular structures induced by the cyclisation. The relatively small Stokes' shift $\left(210 \mathrm{~cm}^{-1}\right.$ for $\left.\mathbf{6} \boldsymbol{\alpha} \boldsymbol{\beta}\right)$ is an extra clear sign of the stiffening of the molecular structure that does not allow large geometrical variation between the ground and the lowest singlet excited states.
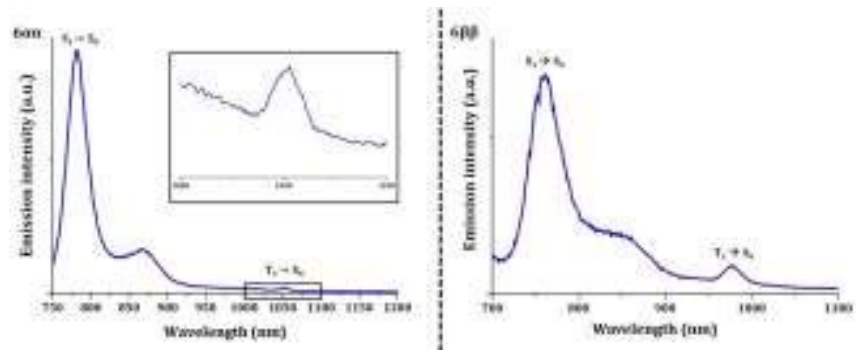

Fig. 5 Emission spectra of compound $\mathbf{6} \boldsymbol{\alpha} \boldsymbol{\alpha}$ (left) and $\mathbf{6} \boldsymbol{\beta} \boldsymbol{\alpha}$ (right) recorded in degassed Me-THF at $-78^{\circ} \mathrm{C}\left(\lambda_{e x}^{6 \alpha \alpha}=745 \mathrm{~nm}, \lambda_{e x}^{6 \beta \alpha}=695 \mathrm{~nm}\right)$.

The other fused compounds being non emissive at room temperature and in aerobic conditions, we performed lowtemperature measurements in degassed Me-THF. Characteristic intense peaks were observed in these experiments (Figure 5) attributed to $S_{1}-S_{0}$ fluorescence at 745 and $780 \mathrm{~nm}$ for $\mathbf{6} \boldsymbol{\alpha} \boldsymbol{\alpha}$ and $\mathbf{6} \boldsymbol{\beta} \boldsymbol{\alpha}$ respectively, whereas we observed additional phosphorescence peaks $\left(\mathrm{T}_{1}-\mathrm{S}_{0}\right)$ at 1055 and $980 \mathrm{~nm}$ respectively with relatively long measured lifetime (11.6 and $13.1 \mu \mathrm{s}$, respectively). In the case of compound $6 \beta \beta$ no fluorescence was observed at low temperature, 
only a phosphorescence peak at $980 \mathrm{~nm}(\tau=17.1 \mu \mathrm{s})$ was detected. The very efficient intersystem crossing observed in the fused system, enhanced by the presence of four sulfur atoms, is therefore obviously one of the main deactivation pathway responsible of the fluorescence extinction at RT in air. We performed DFT calculations of the lowest triplet excited states. The theoretical 0-0 phosphorescence wavelengths determined in THF are $1026 \mathrm{~nm}$ for $\mathbf{6} \boldsymbol{\alpha} \boldsymbol{\alpha}$ and $985 \mathrm{~nm}$ for $\mathbf{6 \beta} \boldsymbol{\beta}$, which are obviously in good agreement with the measurements, hence confirming the above assignments.

As prototypical example of the solid-state absorption of the fused compound, necessary for some optoelectronic applications such as photovoltaics, one can observe, for the $\mathbf{6} \boldsymbol{\alpha} \boldsymbol{\alpha}$, a strong enlargement of the major absorption peak as well as a bathochromic shift compared to the solution (Figure 6). Interestingly, the solid-state absorption is almost panchromatic (no clear-cut transparency range), though with relatively weaker absorption around 450 and $580 \mathrm{~nm}$.

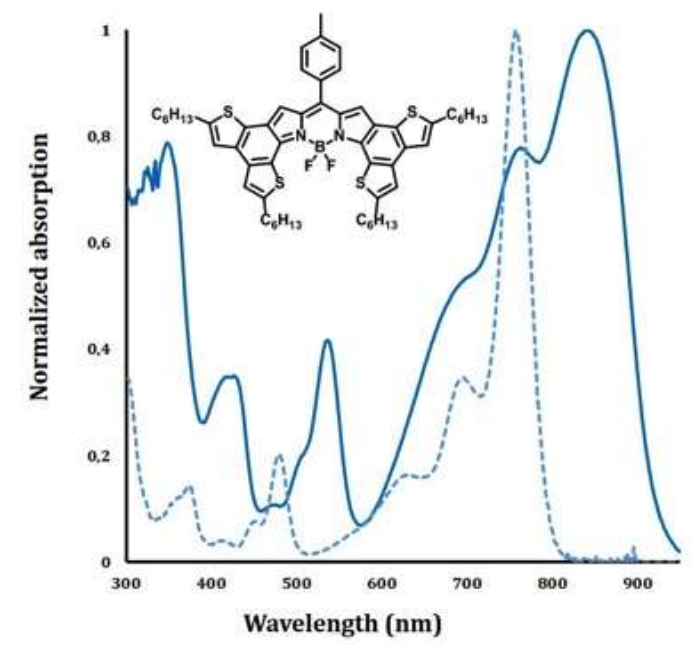

Fig. 6 Absorption of compound $\mathbf{6} \boldsymbol{\alpha} \boldsymbol{\alpha}$ in solution (dotted) and solid state (plain).

\section{Theoretical modeling.}

In order to obtain more insights into the optical properties of these derivatives, we have performed theoretical calculations for the four 6 isomers (replacing the alkyl chains by methyl groups for the sake of computational effort). To this end, we used a protocol, detailed in the SI that combines Time-Dependent Density Functional Theory (TD-DFT) and second-order Coupled-Cluster (CC2). It is indeed wellknown that the former method tends to overestimate the transition energies in fluoroborate and similar derivatives. ${ }^{15}$ Surprisingly, the calculations revealed quite complex and diverse behaviors for the investigated compounds. In all four structures, both TD-DFT and CC2 locate two low-lying excited-states, significantly mixed, though one is dominated by a HOMO-LUMO contribution and the other by a HOMO-1 to LUMO contribution. The results are given in Table S2 in the SI. In $6 \boldsymbol{\alpha} \boldsymbol{\alpha}$ and $\mathbf{6} \boldsymbol{\alpha} \boldsymbol{\beta}$, the former state appears at longer wavelength irrespective of the considered method (TD-DFT or CC2), and according to TD-DFT is very intense, whereas the second appears at significantly higher energy and is much less intense. In
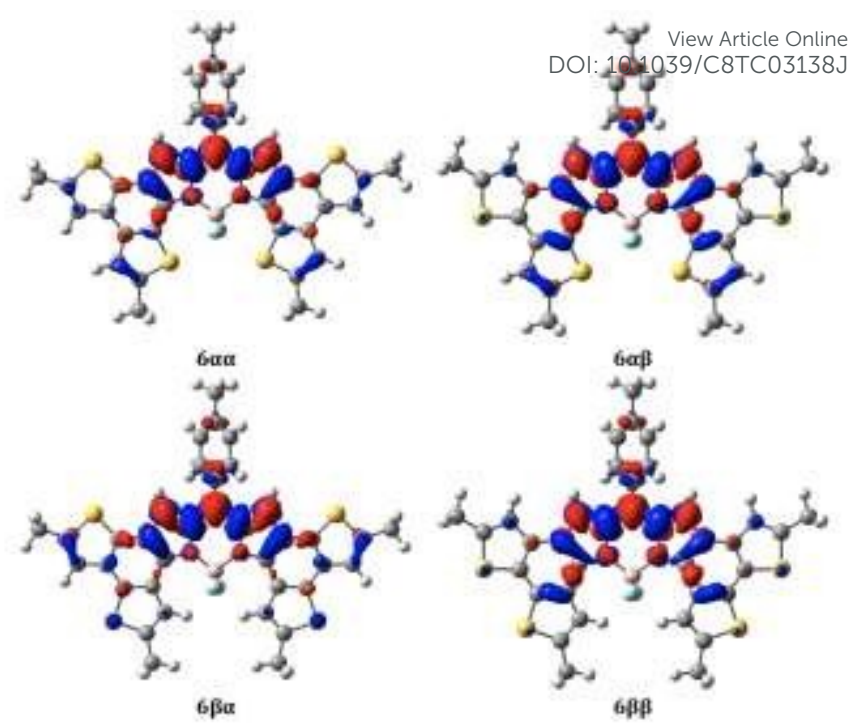

Fig. 7 Electron density difference (EDD) between the excited and ground electronic states for the most intense transition. The red (blue) zones indicate increase (decrease) of density upon electronic transition. Contour threshold: $0.0008 \mathrm{au}$.

$6 \beta \beta$, the two states are predicted to be extremely close energetically (our best estimates are 1.96 and $2.00 \mathrm{eV}$, a difference that is negligible at this level of theory), but only the HOMO-LUMO state presents a significant oscillator strength. In contrast in $6 \beta \alpha$, we foresee that the two states are well separated but with the reverse energetic ordering compared to $6 \alpha \alpha$ and $6 \alpha \beta$ (best estimates of 1.86 and $2.06 \mathrm{eV}$ ) and TD-DFT calculations in toluene predict a significant oscillator strength for the most red-shifted state (0.20). This explains why the typical shape of BODIPY-like spectra with a thin absorption accompanied by a shoulder is totally lost in $\mathbf{6 \beta \boldsymbol { \alpha }}$. For the most intense HOMO-LUMO dominated transition, our theoretical best estimates are $716 \mathrm{~nm}, 677 \mathrm{~nm}, 601$ $\mathrm{nm}$ and $620 \mathrm{~nm}$ for $6 \alpha \boldsymbol{\alpha}, 6 \alpha \beta, 6 \beta \alpha$ and $6 \beta \beta$, respectively. These values can be qualitatively compared to the experimental $]_{\max }$ of $758,719,665$ and $674 \mathrm{~nm}$, respectively. The extra band of $6 \beta \boldsymbol{\alpha}$ is estimated to appear at $666 \mathrm{~nm}$, to be compared to $c a .730 \mathrm{~nm}$, experimentally. Though all theoretical values are blue-shifted compared to experiment, a logical consequence when one neglects the vibronic effects, one clearly notices that the trends are nicely reproduced by the calculations. The electronic density difference (EDD) plots corresponding to the most intense absorption in all dyes are displayed in Figure 7. As expected the core of the molecule acts as a strong acceptor (mostly in red) in all cases, the ring-fused thienyl moieties playing the role of electron donors (in blue in Figure 7). All dyes exhibit very similar excited-state topologies.

\section{Electrochemistry.}

Cyclic voltammetry was used to investigate the oxidation and reduction potential of the fused compounds 6 and thus extrapolate the energy levels of the Highest Occupied Molecular Orbital (HOMO) and the Lowest Unoccupied Molecular Orbital (LUMO) of all compounds 6. All potentials were corrected using the Ferrocene/Ferrocenium couple and assuming its first ionization potential at $-4.78 \mathrm{eV}$ below the vacuum level. ${ }^{16} \mathrm{All}$ of them exhibit perfectly reversible first oxidation and reduction (see Figure 8), allowing the estimation of the ionization potential and the electron 
Table 2 Electrochemical data extracted from the CV measurements. a) EA and IP are estimated from the measured electrochemical onset potentials (vs SCE) as previously ${ }_{I}$ I

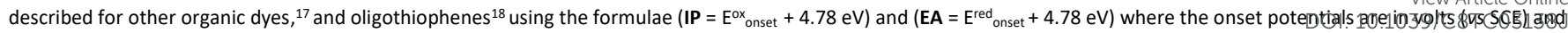
IP and EA are in electron volts. The constant $4.39 \mathrm{eV}$ is the relation between IP, EA and the electrochemical redox potentials which arise from the difference in the gas-phase ionization potential.

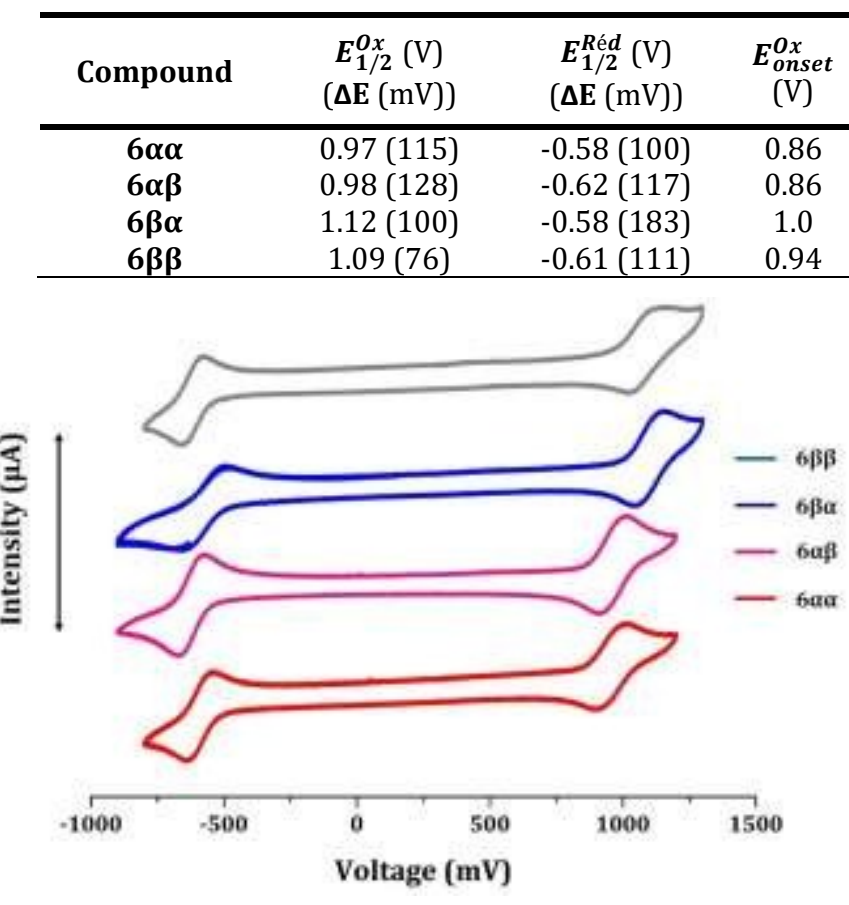

Fig. $8 \mathrm{CV}$ measurements of the four $\alpha$-fused BODIPYs $6 \mathrm{~T}^{1} \mathrm{~T}^{2}$ (recorded in anhydrous dichloromethane at $25^{\circ} \mathrm{C}$ using $\operatorname{TBAPF}_{6}$ as electrolyte).

affinity from the onset value of their oxidation and reduction peaks, respectively.

All of the cyclized products exhibit high electron affinity, with an estimate indicating that their LUMO levels should be very low-lying, around -4.2 to $-4.3 \mathrm{eV}$. Those LUMO values are comparable to the one of PCBM derivatives, widely used as n-type material in bulk heterojunction solar cells. The LUMO level of the cyclized products is almost independent of the structure of the considered $\alpha$-fused BODIPY.

On the isomer series, the electrochemical gap values follow the HOMO levels evolution. Those latter depending themselves on the regioselective anchoring position of the thiophene substituents on the BODIPY core. Indeed, the thiophene connected in $\alpha$-position of the BODIPY core, increases the conjugation and therefore reduced the ionization potential of fused molecule.

\section{Charge transport properties.}

In addition to their promising optical properties, these $\alpha$-fused BODIPY dyes can be expected to present strong intermolecular $\pi-\pi$ interactions in the solid state, which could facilitate the chargecarrier transport. In order to complement the semiconducting properties characterization of those molecules, their charge-carrier mobilities were investigated by using organic field-effect transistor (OFET) devices. The charge transport abilities of the four isomers were first estimated using the bottom-gate/bottom-contact (BGBC) device configuration. The most promising material was next

\begin{tabular}{cccc}
$\begin{array}{c}\boldsymbol{E}_{\text {onset }}^{\text {Red }} \\
(\mathrm{V})\end{array}$ & $\begin{array}{c}\boldsymbol{E}_{\text {HOM⿰ }^{\text {a) }}} \\
(\mathrm{IP})(\mathrm{eV})\end{array}$ & $\begin{array}{c}\boldsymbol{E}_{\boldsymbol{L U M O}} \boldsymbol{a}^{\text {a) }} \\
(\mathrm{EA})(\mathrm{eV})\end{array}$ & $\begin{array}{c}\text { Electrochemical gap } \\
(\mathrm{eV})\end{array}$ \\
\hline-0.52 & -5.64 & -4.26 & 1.38 \\
-0.55 & -5.64 & -4.23 & 1.41 \\
-0.50 & -5.78 & -4.28 & 1.50 \\
-0.54 & -5.72 & -4.24 & 1.48 \\
\hline
\end{tabular}
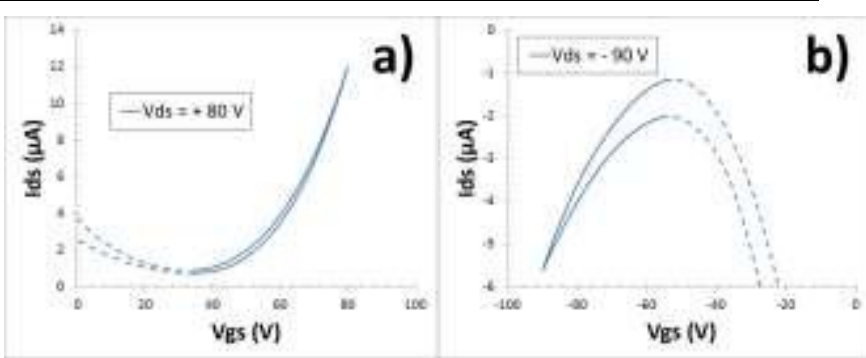

Fig. 9 Transfer characteristics measured on a $6 \alpha \alpha$ top-gate/bottom-contact OFET annealed at $130^{\circ} \mathrm{C}$ for 15 minutes. Figure a) corresponds to the transfer characteristics where the electrons are the main charge-carriers and figure b) to the transfer characteristics where the holes are the main charge-carriers. Ids, Vds and Vgs stands for drain-source current, drain-source voltage and gate-source voltage, respectively.

investigated in top-gate/bottom-contact (TGBC) configuration. The elaboration procedure is detailed in the ESI while the best electron and hole mobilities as extracted in the saturation regime are summarized in the Table 3.

Interestingly, the four isomers display very different behaviors. The two isomers $6 \alpha \boldsymbol{\alpha}$ and $6 \beta \boldsymbol{\alpha}$ show ambipolar charge transport properties while the $6 \boldsymbol{\alpha} \boldsymbol{\beta}$ exhibits only rather moderate hole mobility and the $6 \beta \boldsymbol{\beta}$ presents a really low electron mobility only. The ambipolarity of thienyl-based BODIPY dyes has been already demonstrated and is not surprizing considering the energy levels of those molecules. ${ }^{19}$ The reasons for this charge transport properties evolution in this $\alpha$-fused BODIPY series of molecules, are probably lying in the crystal packing structure of those isomers. For instance, it has been clearly observed that the $\pi$-stacking interactions in the $6 \beta \boldsymbol{\alpha}$ derivative are lower than in the $\mathbf{6} \boldsymbol{\alpha} \boldsymbol{\beta}$ isomer, in-line with the lower mobility values measured for $\mathbf{6} \boldsymbol{\beta} \boldsymbol{\alpha}$. However, given the complexity of such study, this structure-charge transport property relationship is clearly beyond the scope of this paper. Nevertheless, in order to highlight the promising properties of these $\alpha$-fused BODIPY derivatives for optoelectronic applications, we optimized the device configuration, by using a top-gate/bottom-contact transistor with a CYTOP layer as dielectric material. Using the most promising $\mathbf{6} \alpha \boldsymbol{\alpha}$ isomer, well-balanced and high charge-carrier mobilities have been obtained as illustrated by the transfer characteristics displayed in Figure 9.

In the saturation regime, charge-carriers mobilities of $5 \times 10^{-3}$ for holes and $1.4 \times 10^{-2} \mathrm{~cm}^{2} \cdot \mathrm{V}^{-1} \mathrm{~s}^{-1}$ for electrons have been measured demonstrating the great potential of this isomer for application in optoelectronic devices. 
Table 3 Hole $\left(\mu_{\mathrm{h}}\right)$ and electron $\left(\mu_{\mathrm{e}}\right)$ mobilities as calculated from the transfer characteristics of the OFETs in the saturation regime together with the eventual annealing conditions and transistor configuration.

\begin{tabular}{|c|c|c|c|c|}
\hline Compound & $\begin{array}{c}\mu_{\mathrm{h}} \\
\left(\mathrm{cm}^{2} \cdot \mathrm{V}^{-}\right. \\
\left.{ }^{1} \cdot \mathrm{s}^{-1}\right)\end{array}$ & $\begin{array}{c}\boldsymbol{\mu}_{\mathrm{e}} \\
\left(\mathrm{cm}^{2} . \mathrm{V}^{-}\right. \\
\left.{ }^{1} . \mathrm{s}^{-1}\right)\end{array}$ & $\begin{array}{l}\text { Annealing } \\
\left(\min . / T^{\circ} \mathrm{C}\right)\end{array}$ & $\begin{array}{c}\text { OFET } \\
\text { configuration }\end{array}$ \\
\hline $6 \alpha \alpha$ & $5.3 \times 10^{-4}$ & $2.5 \times 10^{-3}$ & $15 / 130$ & BGBC \\
\hline $6 \alpha \beta$ & $5.0 \times 10^{-4}$ & - & $15 / 80$ & BGBC \\
\hline $6 \beta \alpha$ & $3.5 \times 10^{-5}$ & $7.6 \times 10^{-6}$ & - & $B G B C$ \\
\hline $6 \beta \beta$ & - & $1.1 \times 10^{-7}$ & $15 / 80$ & BGBC \\
\hline $6 \alpha \alpha$ & $5.0 \times 10^{-3}$ & $1.4 \times 10^{-2}$ & $15 / 130$ & TGBC \\
\hline
\end{tabular}

\section{Conclusions}

A highly versatile and straightforward synthesis of $\alpha$-fused BODIPY is presented. The synthesis strategy relies on the use of tetrahalogenated BODIPY and on the introduction of four identical aryl groups or two by two different ones (by the use of 3,5-diiodo-2,6dibromo BODIPY) by selective Stille cross-coupling reaction. The aryl groups thus introduced on the dye core can undergo a very efficient oxidative coupling by use of iron(III) chloride to lead to highly planar and conjugated polycyclic dyes. Using this strategy, and by the introduction of thiophene units, we synthesized four regioisomers. X-Ray structure diffraction confirms the high planarity of these isomers. Interestingly, despite similarities, the four isomers display very different optical and electrochemical and electronic properties. These compounds present all an intense absorption (with an extinction coefficient up to $230000 \mathrm{M}^{-1} \mathrm{~cm}^{-1}$ ) in the UV-visible range but with really dissimilar absorption profiles and with absorption onsets ranging from 720 to $800 \mathrm{~nm}$. The different isomers are non or weakly fluorescent probably due to a very efficient intersystem crossing leading to the population of the excited triplet state. Phosphorescence emission could be recorded at 77K confirming this fluorescent non radiative pathway. Despite the fact that all isomers exhibit a high electron affinity, close to the one of soluble fullerene derivatives, they show very different charge transport properties, two molecules display an ambipolar behaviour, while the other two isomers are only hole- and electron-transporting materials, respectively. The best performing isomer, using $\alpha$-thiophenes only, exhibits high and well-balanced charge-carrier mobilities. All those desirable features make this class of BODIPY-based polycyclic dyes attractive and promising organic semiconducting materials for optoelectronic applications. Finally, this very versatile and highly efficient chemical approach allows an endlessly and easy finetuning of the subsequent optoelectronic properties by playing on the aromatic substrates and their regioselective condensations.

\section{Acknowledgements}

View Article Online DOI: 10.1039/C8TC03138J Quentin Huaulmé thanks MENR for PhD fellowship, Nicolas Leclerc and Gilles Ulrich thanks the French National Research Agency (ANR ORION project, ANR-13-PRGE-0001) and the Interreg IV-A program under project C25 Rhin-Solar for their financial support. Denis Jacquemin thanks the GENClCINES/IDRIS, the CCIPL (Centre de Calcul Intensif des Pays de Loire) and a local Troy cluster.

\section{References}

1 G. Ulrich, R. Ziessel and A. Harriman, Angew. Chem. Int. Ed., 2008, 47, 71184.

2 T.G. Pavlopoulos, M. Shah and J.H. Boyer, Appl. Opt., 1988, 27, 4998.

3 E.R. Thapaliya, Y. Zhang, P. Dhakal, A.S. Brown, J.N. Wilson, K.M. Collins and F.M. Raymo, Bioconjug. Chem., 2017, 28 (5), 1519.

4 a) A. Mirloup, N. Leclerc, T. Bura, S. Rihn, R. Bechara, A. Hébraud, P. Lévêque, T. Heiser and R. Ziessel, New J. Chem., 2014, 38, 3644; b) I. Bulut, Q. Huaulmé, A. Mirloup, P. Chávez, S. Fall, A. Hébraud, S. Méry, B. Heinrich, T. Heiser, P. Lévêque and N. Leclerc, ChemSusChem, 2017, 10, 1878.

5 a) C. Jiao, K.-W. Huang and J. Wu, Org. Lett., 2011, 13, 632; b) C. Jiao, L. Zhu and J. Wu, Chem. Eur. J., 2011, 17, 6610.

6 Z. Shen, H. Rohr, K. Rurack, H. Uno, M. Spieles, B. Schulz, G. Reck and N. Ono, Chem. Eur. J., 2004, 10, 4853.

7 a) E. Heyer, P. Retailleau, and R. Ziessel, Org. Lett., 2014, 16, 9, 2330; b) Y. Hayashi, N. Obata, M. Tamaru, S. Yamaguchi, Y. Matsuo, A. Saeki, S. Seki, Y. Kureishi, S. Saito and S. Yamaguchi, Org. Lett. 2012, 14, 866.

8 M.D. Watson, A. Fechtenkotter and K. Müllen, Chem. Rev., 2001, 101, 1267.

9 W. Sheng, Y.-Q. Zheng, Q. Wu, Y. Wu, C. Yu, L. Jiao, E. Hao, J.Y. Wang and J. Pei, Org. Lett., 2017, 19, 2893.

10 L. Jiao, W. Pang, J. Zhou, Y. Wei, X. Mu, G. Bai and E. Hao, J. Org. Chem. 2011, 76, 9988.

11 F. Zeya, J. Lijuan, F. Yuanmei, Y. Changjiang, C. Na, W. Yun, M. Xiaolong, H. Erhong, J. Org. Chem., 2016, 81, 15, 6281.

12 M. J. Ortiz, A. R. Agarrabeitia, G. Duran-Sampedro, J. B. Prieto, T. A. Lopez, W. A. Massad, H. A. Montejano, N. A. García and I. L. Arbeloa, Tetrahedron, 2013, 68, 4, 1153.

13 a) J. Olmsted, J. Phys. Chem., 1979, 83, 2581; b) G. Ulrich, S. Goeb, A. De Nicola, P. Retailleau and R. Ziessel, R. J. Org. Chem., 2011, 76, 4489.

14 A. Poirel, P. Retailleau, A. De Nicola and R. Ziessel, Chem. Eur. J. 2014, 20, 1252.

15 B. Le Guennic and D. Jacquemin, Acc. Chem. Res. 2015, 48, 530.

16 N.G. Connelly and W.E. Geiger, Chem. Rev., 1996, 96, 877.

17 J. Pei, J. Ni, X. Zhou, X. Cao and Y. Lai, J. Org. Chem., 2002, 67, 8104.

18 M. D. Iosip, S. Destri, M. Pasini, W. Porzioc, K. P. Pernstich and B. Batlogg, Synthetic Metals, 2004, 146, 251.

19 T. Bura, N. Leclerc, S. Fall, P. Lévêque, T. Heiser, P. Retailleau, S. Rihn, A. Mirloup and R. Ziessel, J. Am. Chem. Soc., 2012, 134, 17404.

\section{Conflicts of interest}

There are no conflicts to declare. 
A straightforward, highly regioselective and versatile chemical route allows the synthesis of $\alpha$-fused boron dipyrromethene regioisomers.

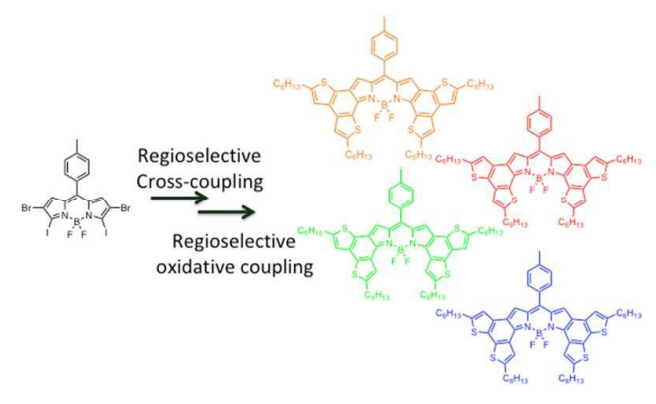

